\title{
MODE-DEPENDENT FINITE-DIFFERENCE DISCRETIZATION OF LINEAR HOMOGENEOUS DIFFERENTIAL EQUATIONS*
}

\author{
C.-C. JAY KUO† AND BERNARD C. LEVY $\ddagger$
}

\begin{abstract}
A new methodology utilizing the spectral analysis of local differential operators is proposed to design and analyze mode-dependent finite-difference schemes for linear homogeneous ordinary and partial differential equations. We interpret the finite-difference method as a procedure for approximating exactly a local differential operator over a finite-dimensional space of test functions called the coincident space, and show that the coincident space is basically determined by the nullspace of the local differential operator. Since local operators are linear and approximately with constant coefficients, we introduce a transform domain approach to perform the spectral analysis. For the case of boundary-value ordinary differential equations (ODEs), a mode-dependent finite-difference scheme can be systematically obtained. For boundaryvalue partial differential equations (PDEs), mode-dependent 5-point, rotated 5-point, and 9-point stencil discretizations for the Laplace, Helmholtz, and convection-diffusion equations are developed. The effectiveness of the resulting schemes is shown analytically, as well as by considering several numerical examples.
\end{abstract}

Key words. convection-diffusion equation, finite-difference method, Helmholtz equation, high-order schemes, Laplace equation, mode-dependent discretization, spectral analysis, transform domain

\section{AMS(MOS) subject classification. 65N05}

1. Introduction. In order to derive a finite-difference approximation for the derivative of a smooth function, a common procedure is to use a Taylor series to expand the function locally and to select the coefficients such that the order of the discretization error is as high as possible. This procedure is based on the assumption that smooth functions can be well approximated by polynomials locally, and in fact it can be shown that the resulting finite-difference approximation is exact for low-order polynomials. However, when the function is exponentially increasing (decreasing) or highly oscillatory, the polynomial representation becomes poor and better finite-difference schemes can be derived by requiring that the derivative of exponential or trigonometric functions should be approximated exactly. In this paper, polynomials and exponential and trigonometric functions are all viewed as modes, and finite-difference schemes obtained by an exact approximation of the derivative of a certain number of modes are called mode-dependent finite-difference schemes. These modes are the coincident modes and the space spanned by them is the coincident space.

Historically, the idea of selecting exponential functions as coincident modes was first suggested by Allen and Southwell [1] for discretizing the convection-diffusion equation. An important feature of this problem is that there are large first-order terms in the governing second-order partial differential equation (PDE). Due to these large first-order terms, there exists a boundary layer that cannot be well approximated by polynomials. The use of trigonometric functions as coincident modes was first discussed by Gautschi [20] for the numerical integration of ordinary differential equations (ODEs) that have periodic or oscillatory solutions whose periods can be estimated in advance.

* Received by the editors March 18, 1987; accepted for publication (in revised form) February 8, 1988. This work was supported in part by Army Research Office grant DAAL03-86-K-0171, in part by the Advanced Research Projects Agency monitored by the Office of Naval Research under contract N000014-81-K-0742, and in part by the Department of Energy under contract DE-FG03-87ER25037, and was performed when the authors were with the Department of Electrical Engineering and Computer Science at the Massachusetts Institute of Technology, Cambridge, Massachusetts 02139.

$\dagger$ Department of Mathematics, University of California, Los Angeles, California 90024.

$\ddagger$ Department of Electrical Engineering and Computer Science, University of California, Davis, California 95616. 
In addition, high-order finite-difference schemes for the Laplace equation were derived by choosing some particular polynomials as coincident modes [33].

Although the concept of a mode-dependent finite-difference discretization procedure has been known for years and mentioned repeatedly in the literature (see for example the references appearing in $\$ 6$ ), few theoretical results about this method have been obtained until now. Important problems, such as whether the mode-dependent finite-difference discretization procedure can always be efficiently applied and how to design such a scheme, remain open. This paper provides a methodology utilizing the spectral analysis of local differential operators to answer these questions. To avoid unnecessary distractions, we will concentrate on one-dimensional and two-dimensional homogeneous boundary-value problems. However, the general methodology described here also applies to initial value problems as well as to nonhomogeneous equations. We will demonstrate this point by referring to some related work.

Since a differential operator is well approximated locally by a linear constantcoefficient operator, the spectral analysis of this local operator becomes relatively easy and a transform domain analysis can be conveniently applied. In the transform domain, the differential and difference operators are algebraic expressions in terms of the complex frequencies $s$ and $z$. We interpret the mode-dependent finite-difference discretization procedure as a way to specify how these two expressions match each other at a certain number of frequencies in the transform domain. This transform domain viewpoint helps us to gain a better understanding of existing mode-dependent finitedifference schemes and serves as a basis for designing new schemes.

We apply the same methodology to both ODEs and PDEs, and develop several mode-dependent finite-difference schemes. The main results include an $(R+1)$-point mode-dependent central difference scheme for an $R$ th-order boundary-value ODE, and 5-point, rotated 5-point, and 9-point stencil discretizations for the two-dimensional Laplace, Helmholtz, and convection-diffusion equations. The mode-dependent finitedifference schemes for the Laplace equation are the same as the conventional ones. However, we present a new derivation. The mode-dependent 5-point and 9-point stencil discretizations of the Helmholtz and convection-diffusion equations are new and have an accuracy proportional to $O\left(h^{2}\right)$ and $O\left(h^{6}\right)$, respectively.

This paper is organized as follows. In $\S 2$, we describe the mode-dependent finite-difference approximation concept in both the space and transform domains. In $\S 3$, we study the discretization of boundary-value ODEs. The problem of determining the coincident space for homogeneous ODEs is discussed and a mode-dependent finite-difference scheme is presented. This scheme is shown to be exact for constantcoefficient ODEs and has a high degree of accuracy for ODEs with smoothly varying coefficients. The extension to the problem of discretizing nonhomogeneous ODEs is briefly addressed. In $\S 4$, we generalize the methodology from one to two dimensions. In particular, we use the Laplace, Helmholtz, and convection-diffusion equations as examples to demonstrate the mode-dependent finite-difference discretization procedure for PDEs. Numerical examples are presented in $\S 5$. In $\S 6$ we discuss several previous related contributions. The main purpose of $\S 6$ is to organize the literature concerning the mode-dependent finite-difference approximation so that more examples will be accessible to interested readers. Some generalizations and concluding remarks are given in $\$ 7$.

2. Mode-dependent finite-difference discretization. Consider the class of functions of the form

$$
u(x)=\sum_{k=1}^{K}\left[c_{k 0}+c_{k 1} x+c_{k 2} \frac{x^{2}}{2 !}+\cdots+c_{k n_{k}} \frac{x^{n_{k}}}{\left(n_{k}\right) !}\right] e^{s_{k} x},
$$


where each term $x^{p} e^{s_{k} x}, 0 \leqq p \leqq n_{k}$, is called a mode of order $p$ at the frequency $s_{k}$. We are interested in approximating a linear $R$ th-order constant-coefficient differential operator operating on $u(x)$,

$$
L(D)=\sum_{r=0}^{R} a_{r} D^{r}
$$

where $D=d / d x$, by an $\left(r_{2}-r_{1}+1\right)$-point finite-difference operator

$$
L_{d}(E)=\sum_{r=r_{1}}^{r_{2}} b_{r} E^{r},
$$

where $E$ is the shift operator defined on a uniform grid $G_{h}$ with spacing $h$, i.e., for $n h,(n+r) h \in G_{h}, E^{r} u(n h)=u((n+r) h) . L_{d}$ corresponds to a forward, backward, or central difference operator depending on whether $r_{1}=0, r_{2}=0$ or $-r_{1}=r_{2}$, respectively. We use

$$
P_{n}(s)=\left\{u(x): u(x)=e^{s x} \sum_{k=0}^{n} c_{k} x^{k}\right\}
$$

to represent the space spanned by polynomials of degree at most $n$ multiplied by the factor $e^{s x}$. A mode-dependent finite-difference discretization scheme is obtained by selecting the coefficients $b_{r}$ of $L_{d}$ such that

$$
\left[L_{d}(E)-L(D)\right] u(x)=0 \text { for } u(x) \in C \text { and } x \in G_{h},
$$

where $C$, called the coincident space of the operator $L_{d}$, is the direct sum of subspaces of the form (2.1), i.e.,

$$
C=\bigoplus_{k=1}^{K} P_{n_{k}}\left(s_{k}\right) .
$$

A mode in the coincident space $C$ is called a coincident mode, and its frequency is called a coincident frequency.

The mode-dependent finite-difference scheme can be conveniently formulated in the transform domain. $L(s)$ is obtained by replacing $D$ with $s$ through the use of the Laplace transform in the $s$-domain,

$$
L(s)=\sum_{r=0}^{R} a_{r} s^{r}
$$

while $L_{d}(z)$ is obtained by replacing $E$ with $z$ through the use of the $Z$-transform in the $z$-domain,

$$
L_{d}(z)=\sum_{r=r_{1}}^{r_{2}} b_{r} z^{r}=\sum_{r=r_{1}}^{r_{2}} b_{r} e^{r s h}
$$

where the last equality is due to the fact that since $E$ is related to $D$ via $E=e^{h D}$ [12], we have $z=e^{s h}$. Then, we can express the difference $\Delta$ between $L$ and $L_{d}$ in terms of a single variable $s$ in the transform domain

$$
\Delta(s)=L_{d}\left(e^{s h}\right)-L(s),
$$

and the characterization (2.2)-(2.3) of the mode-dependent finite-difference scheme can be equivalently stated in the transform domain as

$$
\Delta^{(p)}\left(s_{k}\right)=0, \quad 0 \leqq p \leqq n_{k}, \quad 1 \leqq k \leqq K,
$$


TABLE 1

The approximation of the first-order derivative $D(L(s)=s)$ by a central mode-dependent finite-difference scheme.

\begin{tabular}{ccc}
\hline Modes & $1, e^{\sigma x} \sin (\omega x), e^{\sigma x} \cos (\omega x)$ & $1, e^{\sigma x}, x e^{\sigma x}(\sigma \neq 0)$ \\
\hline$b_{-1}$ & $\frac{e^{\sigma h}[\sigma \sin (\omega h)-\omega \cos (\omega h)]+\omega}{2 \sin (\omega h)[\cos (\omega h)-\cosh (\sigma h)]}$ & $\frac{(1-\sigma h) e^{\sigma h}-1}{2 h[\cosh (\sigma h)-1]}$ \\
$b_{0}$ & $\frac{\omega \cos (\omega h) \sinh (\sigma h)-\sigma \sin (\omega h) \cosh (\sigma h)}{\sin (\omega h)[\cos (\omega h)-\cosh (\sigma h)]}$ & $\frac{\sigma h \cosh (\sigma h)-\sinh (\sigma h)}{h[\cosh (\sigma h)-1]}$ \\
$b_{1}$ & $\frac{e^{-\sigma h}[\sigma \sin (\omega h)+\omega \cos (\omega h)]-\omega}{2 \sin (\omega h)[\cos (\omega h)-\cosh (\sigma h)]}$ & $\frac{-(1+\sigma h) e^{-\sigma h}+1}{2 h[\cosh (\sigma h)-1]}$ \\
\hline Modes & $\frac{1}{2} \frac{1, e^{\sigma_{1} x}, e^{\sigma_{2} x}\left(\sigma_{1} \neq \sigma_{2}\right)}{\sinh \left[\left(\sigma_{2}-\sigma_{1}\right) h\right]+\sinh \left(\sigma_{1} h\right)-\sinh \left(\sigma_{2} h\right)}$ & $\frac{\sigma_{2}\left(1-e^{\sigma_{1} h}\right)-\sigma_{1}\left(1-e^{\sigma_{2} h}\right)}{2 h}$ \\
$b_{-1}$ & $\frac{\sigma_{2} \sinh \left(\sigma_{1} h\right)-\sigma_{1} \sinh \left(\sigma_{2} h\right)}{\sinh \left[\left(\sigma_{2}-\sigma_{1}\right) h\right]+\sinh \left(\sigma_{1} h\right)-\sinh \left(\sigma_{2} h\right)}$ & 0 \\
$b_{0}$ & $\frac{1}{2} \frac{\sigma_{1}\left(1-e^{-\sigma_{2} h}\right)-\sigma_{2}\left(1-e^{-\sigma_{1} h}\right)}{\sinh \left[\left(\sigma_{2}-\sigma_{1}\right) h\right]+\sinh \left(\sigma_{1} h\right)-\sinh \left(\sigma_{2} h\right)}$ & $\frac{1}{2 h}$ \\
$b_{1}$ & &
\end{tabular}

where $\Delta^{(n)}\left(s_{k}\right)=d^{n} \Delta(s) /\left.d s^{n}\right|_{s=s_{k}}$. Let $Q(x)$ be an arbitrary polynomial. Then, the characterization (2.4) is in fact a direct consequence of equalities

$$
L\left(\frac{\partial}{\partial x}\right)\left[Q(x) e^{s x}\right]=L\left(\frac{\partial}{\partial x}\right)\left[Q\left(\frac{\partial}{\partial s}\right) e^{s x}\right]=Q\left(\frac{\partial}{\partial s}\right)\left[L\left(\frac{\partial}{\partial x}\right) e^{s x}\right]=Q\left(\frac{\partial}{\partial s}\right)\left[L(s) e^{s x}\right],
$$

and

$$
L_{d}(E)\left[Q(x) e^{s x}\right]=L_{d}(E)\left[Q\left(\frac{\partial}{\partial s}\right) e^{s x}\right]=Q\left(\frac{\partial}{\partial s}\right)\left[L_{d}(E) e^{s x}\right]=Q\left(\frac{\partial}{\partial s}\right)\left[L_{d}\left(e^{s h}\right) e^{s x}\right] .
$$

It is usually easier to determine the coefficients $b_{r}$ of the mode-dependent finitedifference discretization scheme by applying (2.4) rather than (2.2) and (2.3).

Example 1. To illustrate the mode-dependent discretization procedure described above, the coincident modes and coefficients of several 3-point central difference schemes for the first-order derivative $D$ are listed in Table 1.

\section{Discretization of boundary-value ODEs.}

3.1. Homogeneous ODEs. Consider an $R$ th-order homogeneous 2-point boundary-value problem on $[0,1]$. For convenience, we consider the case $R=2 m$. The case $R=2 m+1$ gives rise to a similar analysis. The equation is written as

$$
L u=0, \quad \text { where } L=\sum_{r=0}^{2 m} a_{r}(x) D^{r} \quad \text { and } \quad a_{2 m}(x)=1,
$$

with given boundary conditions. We discretize (3.1) on a uniform grid with spacing $h$ by a $(2 m+1)$-point central difference scheme,

$$
L_{d} U=0, \quad \text { where } L_{d}=\sum_{r=-m}^{m} b_{r}(n h) E^{r},
$$


and $U$ is the estimate of $u$ on grid points. Suppose that $\phi$ is an arbitrary function in the nullspace $N_{L}$ of operator $L$, and that $N_{L}$ is contained in the coincident space $C$ of $L_{d}$. Then, since

$$
L \phi=0 \quad \text { and } \quad\left[L_{d}-L\right] \phi=0
$$

we obtain

$$
L_{d} \phi=0 \text {. }
$$

Since the discretization for an arbitrary function in the nullspace $N_{L}$ is exact, we conclude that (3.1) is exactly discretized by (3.2).

The nullspace $N_{L}$ is easy to find if the coefficients $a_{r}(x)$ of $L$ are constant. Even if these coefficients are not constant but smoothly varying, $L$ still can be well approximated by a constant-coefficient operator in a local region. This simplification is always assumed for finite-difference schemes since the finite-difference method is a local approximation method. For convenience, we drop the spatial dependency of coefficients $a_{r}(x)$ and $b_{r}(x)$, and use the notation $a_{r} \approx a_{r}\left(x_{0}\right)$ and $b_{r} \approx b_{r}\left(x_{0}\right)$ inside operators $L$ and $L_{d}$ for the rest of this paper. If $a_{r}(x)$ and $b_{r}(x)$ are spatially varying, the discussion is understood to be a local analysis in the neighborhood of $x_{0}$.

The spectral analysis of a linear constant-coefficient operator $L=\sum_{r=0}^{2 m} a_{r} D^{r}$ can be easily performed in the transform domain. We choose the coincident frequencies to be roots of the characteristic equation,

$$
L(s)=s^{2 m}+a_{2 m-1} s^{2 m-1}+\cdots+a_{1} s+a_{0}=0 .
$$

In general, $L(s)$ can be factored as

$$
L(s)=\prod_{k=1}^{K}\left(s-s_{k}\right)^{n_{k}}, \quad \text { where } \sum_{k=1}^{K} n_{k}=2 m,
$$

and $s_{k}$ is known as a natural frequency of $L$ of order $n_{k}$. As a consequence, the operator $L$ has the $2 m$-dimensional nullspace

$$
N_{L}=\bigoplus_{k=1}^{K} P_{n_{k}-1}\left(s_{k}\right) .
$$

A $(2 m+1)$-point finite-difference scheme can be uniquely determined by a $(2 m+$ 1)-dimensional coincident space $C$. However, since a homogeneous finite-difference equation such as (3.2) can be scaled arbitrarily, a $2 m$-dimensional coincident space $C$ is sufficient to specify $L_{d}$ in (3.2). So, letting

$$
C=N_{L} \text {, }
$$

we have an exact discretization scheme for (3.1). For this choice, $L_{d}$ can be determined easily as

$$
L_{d}(z)=A z^{-m} \prod_{k=1}^{K}\left(z-z_{k}\right)^{n_{k}}, \quad \text { where } z_{k}=e^{s_{k} h},
$$

where $A$ is a scaling factor and the multiplication factor $z^{-m}$ is due to the fact that we want $L_{d}(z)$ to be a central difference scheme. This can be verified by substituting $L(s)$ and $L_{d}\left(e^{s h}\right)$ back into (2.4).

Hence, after inverse transformation, we obtain the following mode-dependent finite-difference scheme for (3.1) in the space domain

$$
L_{d} U=0, \quad \text { where } L_{d}(E)=A E^{-m} \prod_{k=1}^{K}\left(E-e^{s_{k} h}\right)^{n_{k}},
$$


and $s_{k}$ is a natural frequency of $L$ of order $n_{k}$. The scaling factor $A$ does not affect the solution of the system of equations (3.5). However, in order to analyze the discretization error $\Delta(s)$ appropriately, it is important to choose $A$ such that $L_{d}\left(e^{s h}\right)$ and $L(s)$ are consistent over fine grids. This consideration requires that the scaling factor $A$ of (3.5) should be proportional to $1 / h^{2 m}$, as $h$ goes to zero.

Example 2. (One-dimensional Laplace equation.) For $L(D)=D^{2}$, we know that $N_{L}=\{1, x\}$. The coincident modes have the same frequency $s_{k}=0$. According to (3.5), we have

$$
L_{d}(E)=A E^{-1}(E-1)^{2}=A\left(E-2+E^{-1}\right) .
$$

If we choose $C=N_{L}+\left\{x^{2}\right\}$, the constant $A$ can be uniquely determined. Solving $L x^{2}=L_{d} x^{2}$, where $x \in G_{h}$, we find that $A=1 / h^{2}$. Then, (3.6) reduces to the standard 3-point central difference scheme.

Example 3. (One-dimensional convection-diffusion equation.) The differential operator is $L(D)=D^{2}-a_{1} D$, where $a_{1} \neq 0$. In this case, $N_{L}=\left\{1, e^{a_{1} x}\right\}$ and $s_{k}=0, a_{1}$. Therefore, by (3.5), we have

$$
L_{d}(E)=A E^{-1}(E-1)\left(E-e^{a_{1} h}\right)=A\left[E-\left(1+e^{a_{1} h}\right)+e^{a_{1} h} E^{-1}\right] .
$$

In particular, if $C=N_{L}+\{x\}$, we find that $A=a_{1} / h\left(e^{a_{1} h}-1\right)$. Then, (3.7) is identical to the scheme considered by Allen and Southwell [1].

For comparison, consider the conventional finite-difference scheme for (3.1),

$$
L_{d, c} U=0, \quad \text { where } L_{d, c}(E)=\sum_{r=0}^{2 m} a_{r} h^{-r} D_{d, 2 m+1}^{r}(E),
$$

where $h^{-r} D_{d, 2 m+1}^{r}(E)$ denotes the $(2 m+1)$-point central difference operator for the $r$ th-order derivative $D^{r}$, which is obtained by selecting $C=P_{2 m}(0)$ as coincident space, and by requiring consistency over fine grids. Then, by comparing (3.5) and (3.8), we see that the mode-dependent scheme (3.5) is obtained by discretizing term by term the product form of the differential operator $L(D)$, whereas the conventional scheme (3.8) is found by discretizing term by term the summation form of $L(D)$.

According to the above discussion, the approximation of the differential operator $L(D)$ in (3.1) by $L_{d}(E)$ given by (3.5) does not give rise to any discretization error when the coefficients $a_{r}$ are constant. This fact is also supported by numerical results.

Of course, the mode-dependent scheme (3.5) gives rise to a discretization error when the coefficients $a_{r}$ are spatially varying. This discretization error depends on the smoothness of the ODE coefficients and the grid size $h$. However, the exact form of this dependency is still unknown, and we have yet to develop a general procedure for estimating the size of the error in this case. In $\S 5$, we use a one-dimensional convectiondiffusion equation as test problem and find that the error of the mode-dependent scheme is proportional to $O\left(\varepsilon h^{2}\right)$, while that of the conventional scheme is proportional to $O\left(h^{2}\right)$, where $\varepsilon$ is the first-order derivative of the coefficient function. The modedependent scheme is always better than the conventional central difference scheme in this test problem and the improvement in accuracy offered by the mode-dependent scheme becomes larger as the coefficient of the convection-diffusion equation becomes smoother.

3.2. Extensions to nonhomogeneous ODEs. Suppose that (3.1) includes a driving function $f(x)$, so that

$$
L u-f=0 .
$$


By performing a Taylor series expansion of $f(x)$ in the vicinity of a discretization point $x_{0}$, we can assume that $f$ is approximated locally by a polynomial of low degree, i.e.,

$$
f(x) \approx c_{0}+c_{1} x+c_{2} x^{2}+\cdots .
$$

A general discretization scheme for (3.9), which has been proposed in the context of the OCI and HODIE methods [4], [7], [32], is

$$
L_{d} U-I_{d} f=L_{d} U-I_{d} L u=0,
$$

where $I_{d}$ is an averaging operator.

The set of functions whose images through $L$ are polynomials of degree less or equal to $l$ defines the space

$$
P_{L, l}=\left\{u(x): L u=\sum_{r=0}^{l} p_{r} x^{r}\right\} .
$$

Note that since the coefficients $p_{r}$ above can all be selected equal to zero, $N_{L}$ is also included in $P_{L, l}$. The space $P_{L, l}$ will be used here to approximate the solution space of (3.9). Suppose that $\psi$ is an arbitrary function of the space $P_{L, l}$. Ideally, we want

$$
L_{d} \psi-I_{d} L \psi=0,
$$

in order to guarantee that the discretization (3.10) of the nonhomogeneous equation (3.9) is exact in the approximated solution space $P_{L, l}$.

In particular, if $I_{d}$ is chosen to be the identity operator $I$, (3.11) becomes

$$
\left(L_{d}-L\right) \psi=0 .
$$

Therefore, the coincident space $C$ of the finite-difference operator $L_{d}$ for the nonhomogeneous equation (3.9) has to be

$$
C=P_{L, l} \text {. }
$$

The major disadvantage of this choice is that the dimension of $C$ is larger than that of $N_{L}$. Hence, a finite-difference method with more than $(2 m+1)$ points will be necessary and more computations will be required.

The purpose of introducing $I_{d}$ is to reduce the dimension of the coincident space. For a $(2 m+1)$-point finite-difference scheme, we can decompose the discretization scheme (3.10) into two steps. First, by choosing $C=P_{L, 0}$, we can uniquely determine $L_{d}$. Then, by using an arbitrary function $\psi$ of the space $P_{L, I} \ominus P_{L, 0}$ as test function for (3.11), we can solve for the coefficients of $I_{d}$. This procedure is illustrated by the following example.

Example 4. (One-dimensional Poisson equation.) In this case, we have $L(D)=D^{2}$, $N_{L}=\{1, x\}$, and $P_{L, l}=\left\{1, x, x^{2}, x^{3}, \cdots, x^{l+2}\right\}$. By choosing $C=P_{L, 0}$, we know from Example 2 that

$$
L_{d}(E)=\frac{1}{h^{2}}\left(E-2+E^{-1}\right) .
$$

Assuming that $I_{d}(E)=d_{-1} E^{-1}+d_{0}+d_{1} E$ with respect to the same grid $G_{h}$ and solving (3.11) with $L_{d}$ given above and $\psi=x^{3}, x^{4}$, we obtain

$$
I_{d}(E)=\frac{1}{12} E^{-1}+\frac{5}{6}+\frac{1}{12} E \text {. }
$$

Then, for this case, the discretization (3.10) corresponds to the classical StormerNumerov approximation, and it is exact for any function in the space $P_{L, 2}$. More generally, we call $P_{L, l}$ the generalized coincident space $C_{g}\left(L_{d}, I_{d}\right)$ for the approximation 
(3.10) of (3.9). Note that the dimension of $C_{g}$ for the above example is 5, and that there are five independent parameters in $\left(L_{d}, I_{d}\right)$ since $(3.11)$ can be scaled by an arbitrary constant.

The above approach is different from the HODIE method. For an $R$ th-order nonhomogeneous ODE, the HODIE method uses polynomials of degree less than or equal to $n$, i.e., $P_{n}(0)$ with $n>R$ as the generalized coincident space $C_{g}$ for (3.10). It does not exploit any special structure of the differential operator $L$. In contrast, our mode-dependent method uses the approximated solution space $P_{L, n-R}$ as the generalized coincident space $C_{g}$. Hence, a spectral analysis of the operator $L$ is necessary. In particular, when $L=D^{R}, P_{L, n-R}$ is the same as $P_{n}(0)$. Then, there is no difference between the HODIE and mode-dependent methods.

The determination of the averaging operator $I_{d}$ for the HODIE method has been discussed in detail [7], [32]. For example, the operator $I_{d}$ may be defined on an auxiliary grid different from the discretization grid $G_{h}$. A similar approach can also be used to design $I_{d}$ for the mode-dependent method. Note that the selection of the averaging operator $I_{d}$ has no effect on functions in the nullspace $N_{L}$. Therefore, the coincident space $C$ of $L_{d}$ has to contain $N_{L}$ so that the discretization error for functions in $N_{L}$ can be eliminated by choosing an appropriate $L_{d}$.

In this paper, we focus primarily on the determination of the coincident space $C$ and of the finite-difference operator $L_{d}$. In the next section, we will therefore restrict our attention to homogeneous boundary-value PDEs and we will attempt to extend the methodology developed in this section to the discretization of this specific class of PDE problems.

4. Discretization of boundary-value PDEs. Consider a general two-dimensional boundary-value PDE on the square $[0,1]^{2}$

$$
L\left(D_{x}, D_{y}\right) u=0, \quad \text { where } L\left(D_{x}, D_{y}\right)=\sum_{r, s} a_{r, s} D_{x}^{r} D_{y}^{s},
$$

with $D_{x}^{r}=\partial^{r} / \partial x^{r}$ and $D_{y}^{s}=\partial^{s} / \partial y^{s}$, and with appropriate boundary conditions. We discretize (4.1) with the finite-difference scheme

$$
L_{d}\left(E_{x}, E_{y}\right) U=0 \text {, where } L\left(E_{x}, E_{y}\right)=\sum_{r, s} b_{r, s} E_{x}^{r} E_{y}^{s},
$$

and where $E_{x}$ and $E_{y}$ are, respectively, the shift operators in the $x$ - and $y$-directions on the grid $G_{h_{x}, h_{y}}$. Relying on a natural generalization of the one-dimensional case, we have the following associations between the two-dimensional space domain operators and transform domain variables:

$$
D_{x} \leftrightarrow s_{x}, \quad D_{y} \leftrightarrow s_{y}, \quad E_{x} \leftrightarrow z_{x}, \quad E_{y} \leftrightarrow z_{y},
$$

where $s_{x}=\sigma_{x}+i \omega_{x}$ and $s_{y}=\sigma_{y}+i \omega_{y}$. They are related via $E_{x}=e^{h_{x} D_{x}}, E_{y}=e^{h_{y} D_{y}}, z_{x}=$ $e^{h_{x} s_{x}}$, and $z_{y}=e^{h_{y} s_{y}}$. To simplify the following discussion, we will only consider the case $h_{x}=h_{y}=h$.

Substituting $e^{s_{x} x+s_{y} y}$ inside (4.1), we obtain the characteristic equation

$$
\sum_{r, s} a_{r, s} s_{x}^{r} s_{y}^{s}=0 .
$$

There are two complex variables in (4.3), but since we have only one (complex) equation, there are infinitely many solutions to this equation and therefore infinitely many modes in $N_{L}$. It is not possible to approximate all modes in $N_{L}$ exactly. Thus, we have to select a finite-dimensional subspace $D_{L} \subset N_{L}$, called the dominant-mode space, as the coincident space $C$ for $L_{d}$. The determination of $D_{L}$ depends on a rough 
estimation of the local behavior of the solution. This information is usually provided by the structure of the PDE operator and the corresponding boundary conditions. In this section, we restrict our attention to the case where the dominant modes are either oscillating or exponentially growing (decaying). In other words, coincident frequencies are selected among the sets

$$
\left\{\left(s_{x}, s_{y}\right):\left(s_{x}, s_{y}\right)=\left(\sigma_{x}, \sigma_{y}\right)\right\} \quad \text { or } \quad\left\{\left(s_{x}, s_{y}\right):\left(s_{x}, s_{y}\right)=\left(i \omega_{x}, i \omega_{y}\right)\right\} .
$$

We do not consider complex coincident frequencies, since they generally lead to discretization schemes with complex coefficients which complicate the solution procedure. However, even under (4.4), the mode-dependent concept still does not lead to a unique discretization scheme. By taking into account the symmetrical property of the spectra of the differential and difference operators and the solubility of the resulting finite-difference schemes, we can further constrain ourselves within a much smaller design space. In the following, the 5-point, rotated 5-point, and 9-point stencil discretizations for the Laplace, Helmholtz, and convection-diffusion equations will be used as examples to demonstrate the mode-dependent discretization concept.

4.1. Laplace equation. For the Laplace equation, we have $L\left(D_{x}, D_{y}\right)=D_{x}^{2}+D_{y}^{2}$. Since only one frequency $\left(s_{x}, s_{y}\right)=(0,0)$ satisfies the characteristic equation and belongs to the sets $(4.4)$ of interest, $(0,0)$ is selected as the unique coincident frequency. In this case, the mode-dependent scheme is the same as the conventional scheme.

The following 5-point, rotated 5-point, and 9-point stencil discretization schemes have been derived by several approaches [12], [26], [33]:

$$
\begin{aligned}
& L_{d,+}\left(E_{x}, E_{y}\right)=\frac{1}{h^{2}}\left(E_{x}+E_{x}^{-1}+E_{y}+E_{y}^{-1}-4\right), \\
& L_{d, \times}\left(E_{x}, E_{y}\right)=\frac{1}{2 h^{2}}\left(E_{x} E_{y}+E_{x}^{-1} E_{y}+E_{x} E_{y}^{-1}+E_{x}^{-1} E_{y}^{-1}-4\right), \\
& L_{d, 9}=\frac{1}{6 h^{2}}\left[4\left(E_{x}+E_{x}^{-1}+E_{y}+E_{y}^{-1}\right)+\left(E_{x} E_{y}+E_{x}^{-1} E_{y}+E_{x} E_{y}^{-1}+E_{x}^{-1} E_{y}^{-1}\right)-20\right] .
\end{aligned}
$$

It is well known that they have, respectively, an accuracy of $O\left(h^{2}\right), O\left(h^{2}\right)$, and $O\left(h^{6}\right)$ when used to discretize the Laplace equation [26].

Here, we present another derivation of these schemes by matching $L\left(s_{x}, s_{y}\right)$ and $L_{d}\left(z_{x}, z_{y}\right)$ at the coincident frequency $(0,0)$ in the transform domain. As before, we consider the expansion of $\Delta=L_{d}-L$ around $(0,0)$,

$$
\begin{aligned}
& \Delta\left(s_{x}, s_{y}\right)=\Delta^{(0,0)}(0,0)+\Delta^{(1,0)}(0,0) s_{x}+\Delta^{(0,1)}(0,0) s_{y} \\
&+\frac{1}{2}\left[\Delta^{(2,0)}(0,0) s_{x}^{2}+\Delta^{(1,1)}(0,0) 2 s_{x} s_{y}+\Delta^{(0,2)}(0,0) s_{y}^{2}\right] \\
&+\sum_{\substack{p+q \geqq 3 \\
p, q \geqq 0}} \Delta^{(p, q)}(0,0) \frac{1}{p ! q !} s_{x}^{p} s_{y}^{q},
\end{aligned}
$$

where

$$
\Delta^{(p, q)}(0,0)=\left.\frac{\partial^{p+q} \Delta\left(s_{x}, s_{y}\right)}{\partial^{p} s_{x} \partial^{q} s_{y}}\right|_{\left(s_{x}, s_{y}\right)}=(0,0),
$$

which is a function of the grid size $h$. Hence, (4.8) is in fact a power series of $h$. Our derivation attempts to make the order of the residual terms in (4.8) as high as possible. 
The discretization schemes (4.5) and (4.6) can be derived by requiring, respectively, that

$$
\Delta^{(0,0)}(0,0)=\Delta^{(1,0)}(0,0)=\Delta^{(0,1)}(0,0)=\Delta^{(2,0)}(0,0)=\Delta^{(0,2)}(0,0)=0,
$$

and

$$
\Delta^{(0,0)}(0,0)=\Delta^{(1,0)}(0,0)=\Delta^{(0,1)}(0,0)=\Delta^{(1,1)}(0,0)=\Delta^{(2,0)}(0,0)=\Delta^{(0,2)}(0,0)=0 .
$$

Note the similarity between the above requirements and (2.4). The above choice of constraints $\Delta^{(p, q)}(0,0)=0$ has taken into account the specific structure of operators $L_{d,+}, L_{d, \times}$, and $L$. For example, in the case of $L_{d, \times}$, the symmetry properties of $L_{d, \times}$ imply that $\Delta^{(2,0)}(0,0)=\Delta^{(0,2)}(0,0)$, so that among the six constraints that are used to specify $L_{d, \times}\left(E_{x}, E_{y}\right)$, only five are independent.

By setting the coefficients of low-order terms in (4.8) equal to zero, it is possible to obtain various high-order finite-difference discretization schemes. For example, to obtain the 9-point scheme (4.7), we need only to impose the requirement that this scheme should have an accuracy of $O\left(h^{6}\right)$ for modes satisfying the characteristic equation $s_{x}^{2}+s_{y}^{2}=0$. Then, substituting the identity $s_{x}^{2}+s_{y}^{2}=0$ inside (4.8) and setting coefficients up to order $h^{5}$ equal to zero, we obtain nine independent constraints that specify (4.7) uniquely.

4.2. Helmholtz equation. For the Helmholtz equation, we have

$$
L\left(D_{x}, D_{y}\right)=D_{x}^{2}+D_{y}^{2}+\lambda^{2} \text {. }
$$

If $s_{x}$ and $s_{y}$ are purely imaginary, the characteristic equation becomes

$$
\omega_{x}^{2}+\omega_{y}^{2}=\lambda^{2}
$$

which is a circle in the $\omega_{x}-\omega_{y}$ plane, centered at the origin and with radius $|\lambda|$. There are infinitely many natural frequencies and, hence, there are many different ways to select coincident frequencies. In this section, we design mode-dependent 5-point, rotated 5-point, and 9-point stencil discretization schemes based on the following two considerations. First, if there is no further information about the dominant modes, a reasonable strategy is to distribute coincident frequencies uniformly along the contour (4.9). Second, we want to preserve the symmetry properties of $L$ so that the resulting discretization scheme is in a simple form and can easily be implemented.

Let us select

$$
\left(\omega_{x}, \omega_{y}\right)=\left(|\lambda| \cos \left(\frac{n}{2} \pi+\frac{1}{4} \pi\right),|\lambda| \sin \left(\frac{n}{2} \pi+\frac{1}{4} \pi\right)\right), \quad 0 \leqq n \leqq 3,
$$

as coincident frequencies as shown in Fig. 1(a). With this choice, the discretization along the $x$ - and $y$-directions can be treated independently. The resulting scheme is

$$
L_{d}\left(E_{x}, E_{y}\right)=A\left[E_{x}^{-1}-2 \cos \left(\frac{|\lambda|}{\sqrt{2}} h\right)+E_{x}+\kappa\left(E_{y}^{-1}-2 \cos \left(\frac{|\lambda|}{\sqrt{2}} h\right)+E_{y}\right)\right] \text {. }
$$

Two parameters $A$ and $\kappa$ remain undetermined in the above expression. The parameter $\kappa$ is selected such that the discretization error $\Delta\left(s_{x}, s_{y}\right)$ at natural frequencies is proportional to $O\left(h^{2}\right)$, and the parameter $A$ is used to normalize the above scheme so that $L_{d}$ is consistent with $L$. A simple choice of $\kappa$ and $A$ for the Helmholtz equation is $\kappa=1$ and $A=1 / h^{2}$. Hence, this gives a symmetrical 5-point stencil discretization operator

$$
L_{d,+}\left(E_{x}, E_{y}\right)=\frac{1}{h^{2}}\left[E_{x}^{-1}+E_{x}+E_{y}^{-1}+E_{y}-4 \cos \left(\frac{|\lambda|}{\sqrt{2}} h\right)\right] .
$$




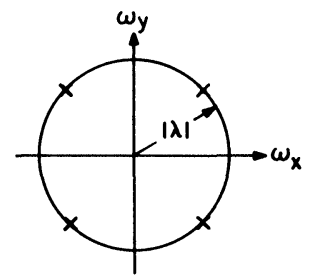

(a)

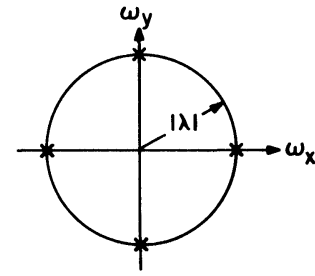

(b)

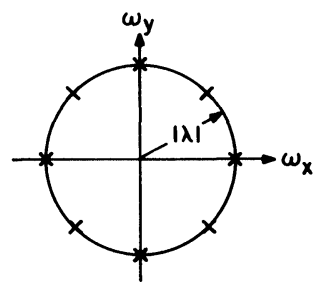

(c)

FIG. 1. Coincident frequencies of the mode-dependent (a) 5-point, (b) rotated 5-point, and (c) 9-point stencil discretizations of the Helmholtz equation.

Rotating the above four coincident frequencies in the transform domain and the above 5 -point stencil in the space domain by an angle $\frac{1}{4} \pi$, we obtain another mode-dependent 5-point stencil discretization. In this scheme, the coincident frequencies become

$$
\left(\omega_{x}, \omega_{y}\right)=\left(|\lambda| \cos \left(\frac{n}{2} \pi\right),|\lambda| \sin \left(\frac{n}{2} \pi\right)\right), \quad 0 \leqq n \leqq 3
$$

as shown in Fig. 1(b), and the resulting 5-point stencil operator is

$$
L_{d, \times}\left(E_{x}, E_{y}\right)=\frac{1}{2 h^{2}}\left[E_{x}^{-1} E_{y}^{-1}+E_{x}^{-1} E_{y}+E_{x} E_{y}^{-1}+E_{x} E_{y}-4 \cos (|\lambda| h)\right] .
$$

Notice that this rotated 5-point stencil can be viewed as corresponding to a discretization scheme on a grid with spacing $\sqrt{2} h$. By appropriately combining (4.10), (4.11), and adding a constant term, we obtain the 9-point stencil discretization operator

$$
L_{d, 9}\left(E_{x}, E_{y}\right)=\frac{\gamma_{\times}}{\gamma_{\times}+\gamma_{+}} L_{d,+}\left(E_{x}, E_{y}\right)+\frac{\gamma_{+}}{\gamma_{\times}+\gamma_{+}} L_{d, \times}\left(E_{x}, E_{y}\right)-\frac{\gamma_{\times} \gamma_{+}}{\gamma_{\times}+\gamma_{+}} .
$$

Then, if

$$
\begin{aligned}
& \gamma_{\times}=L_{d, \times}\left(e^{i(|\lambda| / \sqrt{2}) h}, e^{i(|\lambda| / \sqrt{2}) h}\right)=\frac{1}{h^{2}}[\cos (\sqrt{2}|\lambda| h)+1-2 \cos (|\lambda| h)], \\
& \gamma_{+}=L_{d,+}\left(e^{i|\lambda| h}, 1\right)=\frac{1}{h^{2}}\left[2 \cos (|\lambda| h)+2-4 \cos \left(\frac{|\lambda|}{\sqrt{2}} h\right)\right],
\end{aligned}
$$


we are able to match $L_{d}\left(z_{x}, z_{y}\right)$ and $L\left(s_{x}, s_{y}\right)$ at eight frequencies

$$
\left(\omega_{x}, \omega_{y}\right)=\left(|\lambda| \cos \left(\frac{n}{4} \pi\right),|\lambda| \sin \left(\frac{n}{4} \pi\right)\right), \quad 0 \leqq n \leqq 7
$$

as shown in Fig. 1(c). Thus, (4.12) is the desired mode-dependent 9-point stencil discretization operator.

When $|\lambda|$ goes to zero, the Helmholtz equation reduces to the Laplace equation and schemes (4.10)-(4.12) converge to (4.5)-(4.7). So, schemes (4.10)-(4.12) can be viewed as a natural generalization of (4.5)-(4.7) and apply to both $\lambda=0$ and $\lambda \neq 0$. The error estimate of the above schemes for the test functions $e^{s_{x} x+s_{y} y}$, where $s_{x}$ and $s_{y}$ satisfy the characteristic equation $s_{x}^{2}+s_{y}^{2}+\lambda^{2}=0$, can be obtained straightforwardly. Since

$$
\Delta\left(D_{x}, D_{y}\right) e^{s_{x} x+s_{y} y}=L_{d}\left(e^{D_{x} h}, e^{D_{y} h}\right) e^{s_{x} x+s_{y} y}=L_{d}\left(e^{s_{x} h}, e^{s_{y} h}\right) e^{s_{x} x+s_{y} y},
$$

we only have to replace $E_{x}$ and $E_{y}$ with $e^{s_{x} h}$ and $e^{s_{y} h}$ inside $L_{d}\left(E_{x}, E_{y}\right)$ and use a Taylor series expansion to simplify the resulting algebraic equation. By using this approach, we find that

$$
\begin{aligned}
& L_{d,+}\left(e^{s_{x} h}, e^{s_{y} h}\right)=\left(\frac{1}{24} \lambda^{4}-\frac{1}{6} s_{x}^{2} s_{y}^{2}\right) h^{2}+\left(\frac{-1}{480} \lambda^{6}+\frac{1}{120} \lambda^{2} s_{x}^{2} s_{y}^{2}\right) h^{4}+O\left(h^{6}\right), \\
& L_{d, \times}\left(e^{s_{x} h}, e^{s_{y} h}\right)=\frac{1}{3} s_{x}^{2} s_{y}^{2} h^{2}-\frac{1}{30} \lambda^{2} s_{x}^{2} s_{y}^{2} h^{4}+O\left(h^{6}\right) .
\end{aligned}
$$

Since $\gamma_{+}=L_{d,+}\left(e^{i|\lambda| h}, 1\right)$ and $\gamma_{\times}=L_{d, \times}\left(e^{i(|\lambda| / \sqrt{2}) h}, e^{i(|\lambda| / \sqrt{2}) h}\right)$, we also have

$$
\gamma_{+}=\frac{1}{24} \lambda^{4} h^{2}-\frac{1}{480} \lambda^{6} h^{4}+O\left(h^{6}\right), \quad \gamma_{\times}=\frac{1}{12} \lambda^{4} h^{2}-\frac{1}{120} \lambda^{6} h^{2}+O\left(h^{6}\right) .
$$

Combining (4.13)-(4.15), we have $\gamma_{\times}+\gamma_{+}=O\left(h^{2}\right)$ and

$$
\left(\gamma_{\times}+\gamma_{+}\right) L_{d, 9}\left(e^{s_{x} h}, e^{s_{y} h}\right)=\gamma_{\times} L_{d,+}\left(e^{s_{x} h}, e^{s_{y} h}\right)+\gamma_{+} L_{d, \times}\left(e^{s_{x} h}, e^{s_{y} h}\right)-\gamma_{\times} \gamma_{+}=O\left(h^{8}\right) .
$$

We know that both $L_{d,+}$ and $L_{d, \times}$ have an accuracy of $O\left(h^{2}\right)$ from (4.13) and (4.14), and that $L_{d, 9}$ has an accuracy of $O\left(h^{6}\right)$ from (4.16). Note also that since the coefficients in front of $h^{2}$ in (4.13) and (4.14) are functions of $\lambda, s_{x}$, and $s_{y}$ for a fixed value of $h$, the above discretization schemes are less accurate when $|\lambda|$ is large.

Unlike in the ODE case, the mode-dependent schemes for PDEs cannot catch all modes in the nullspace of $L$, so that there exist discretization errors even for constantcoefficient PDEs. Rigorously speaking, the above error estimate applies only to constant-coefficient PDEs. If the coefficients of the PDE of interest are spatially varying, the error associated to mode-dependent schemes is still unknown. But, we suspect that when the coefficients are smoothly varying, the error is approximately the same as for constant coefficients.

Conventional finite-difference schemes for the Helmholtz equation are derived by discretizing the Laplacian with operators (4.5)-(4.7) and then combining them with the remaining term $\lambda^{2} u$. The resulting schemes all have an accuracy of $O\left(h^{2}\right)$. Therefore, the conventional 9-point discretization scheme is much worse than the mode-dependent 9-point scheme. Although the conventional and mode-dependent 5-point schemes have the same order of accuracy, the mode-dependent schemes (4.10) and (4.11) are more accurate than conventional schemes along the contour (4.9). To show this, the discretization errors for mode-dependent and conventional 5-point discretization schemes are plotted in Fig. 2 for the case where $\lambda=10$ and $h=0.1$.

On the other hand, we can also consider the discretization of the operator

$$
\tilde{L}\left(D_{x}, D_{y}\right)=D_{x}^{2}+D_{y}^{2}-\lambda^{2} \text {. }
$$




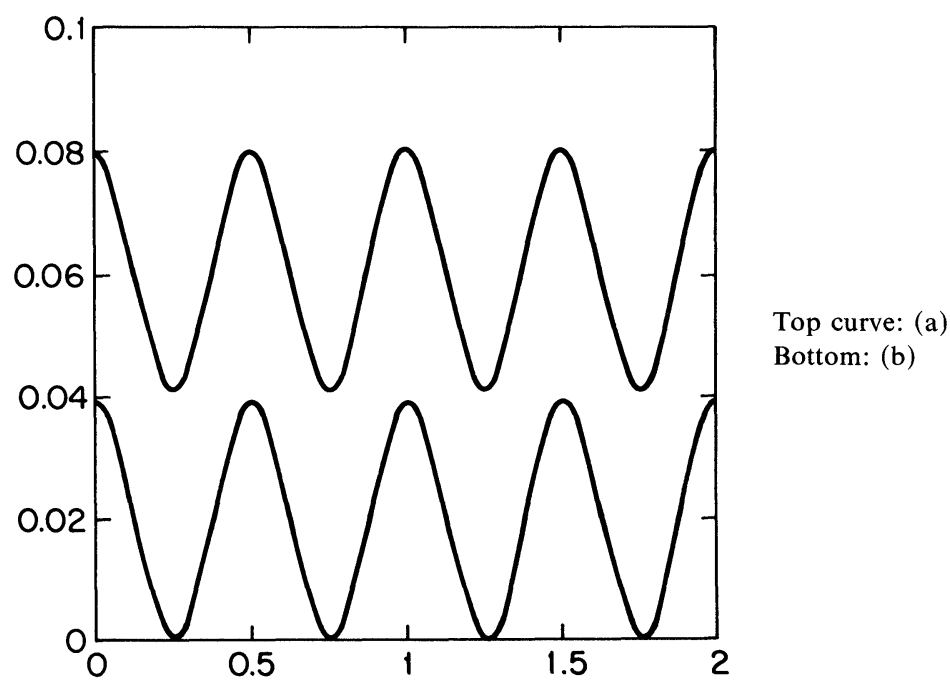

FIG. 2. Plot of $\left|\Delta\left(\omega_{x}, \omega_{y}\right)\right|$ as a function of $c$ along the contour $\left(\omega_{x}, \omega_{y}\right)=(|\lambda| \cos (c \pi),|\lambda| \sin (c \pi))$, for (a) conventional and (b) mode-dependent 5-point stencil discretizations of the Helmholtz equation.

Considering only the real frequencies $\left(s_{x}, s_{y}\right)=\left(\sigma_{x}, \sigma_{y}\right)$, we have the characteristic equation

$$
\sigma_{x}^{2}+\sigma_{y}^{2}=\lambda^{2}
$$

Thus, for the present case, we examine the $\sigma_{x}-\sigma_{y}$ plane, instead of the $\omega_{x}-\omega_{y}$ plane. By using an approach similar to the one described above, we get the following 5-point, rotated 5-point, and 9-point stencil discretization schemes:

$$
\begin{aligned}
& \tilde{L}_{d,+}\left(E_{x}, E_{y}\right)=\frac{1}{h^{2}}\left[E_{x}^{-1}+E_{x}+E_{y}^{-1}+E_{y}-4 \cosh \left(\frac{|\lambda|}{\sqrt{2}} h\right)\right] \\
& \tilde{L}_{d, \times}\left(E_{x}, E_{y}\right)=\frac{1}{2 h^{2}}\left[E_{x}^{-1} E_{y}^{-1}+E_{x}^{-1} E_{y}+E_{x} E_{y}^{-1}+E_{x} E_{y}-4 \cosh (|\lambda| h)\right], \\
& \tilde{L}_{d, 9}\left(E_{x}, E_{y}\right)=\frac{\gamma_{\times}}{\gamma_{\times}+\gamma_{+}} \tilde{L}_{d,+}\left(E_{x}, E_{y}\right)+\frac{\gamma_{+}}{\gamma_{\times}+\gamma_{+}} \tilde{L}_{d, \times}\left(E_{x}, E_{y}\right)-\frac{\gamma_{\times} \gamma_{+}}{\gamma_{\times}+\gamma_{+}}
\end{aligned}
$$

where

$$
\begin{aligned}
& \gamma_{\times}=\frac{1}{h^{2}}[\cosh (\sqrt{2}|\lambda| h)+1-2 \cosh (|\lambda| h)], \\
& \gamma_{+}=\frac{1}{h^{2}}\left[2 \cosh (|\lambda| h)+2-4 \cosh \left(\frac{|\lambda|}{\sqrt{2}} h\right)\right] .
\end{aligned}
$$

These schemes have an accuracy of $O\left(h^{2}\right), O\left(h^{2}\right)$, and $O\left(h^{6}\right)$, respectively.

4.3. Convection-diffusion equation. For the convection-diffusion equation, the differential operator takes the form

$$
L\left(D_{x}, D_{y}\right)=D_{x}^{2}+D_{y}^{2}-2 \alpha D_{x}-2 \beta D_{y} .
$$


In particular, if we consider only real frequencies $\left(s_{x}, s_{y}\right)=\left(\sigma_{x}, \sigma_{y}\right)$, the corresponding characteristic equation is

$$
\sigma_{x}^{2}+\sigma_{y}^{2}-2 \alpha \sigma_{x}-2 \beta \sigma_{y}=0,
$$

which is a circle in the $\sigma_{x}-\sigma_{y}$ plane centered at $(\alpha, \beta)$ with radius $d=\sqrt{\alpha^{2}+\beta^{2}}$.

The conventional approach for discretizing the above equation relies on a central difference scheme to approximate the first- and second-order derivatives separately. This gives

$$
L_{d, c}\left(E_{x}, E_{y}\right)=\frac{1}{h^{2}}\left[(1+\alpha h) E_{x}^{-1}+(1-\alpha h) E_{x}-4+(1+\beta h) E_{y}^{-1}+(1-\beta h) E_{y}\right],
$$

which corresponds to selecting a single coincident frequency at the origin. Allen and Southwell [1] combined two one-dimensional mode-dependent schemes along the $x$ and $y$-directions (also see Example 3 in $\S 3$ ). This leads to

$$
\begin{aligned}
L_{d, A S}\left(E_{x}, E_{y}\right)=\frac{1}{h}[ & \frac{2 \alpha}{e^{2 \alpha h}-1}\left(e^{2 \alpha h} E_{x}^{-1}-1-e^{2 \alpha h}+E_{x}\right) \\
& \left.+\frac{2 \beta}{e^{2 \beta h}-1}\left(e^{2 \beta h} E_{y}^{-1}-1-e^{2 \beta h}+E_{y}\right)\right],
\end{aligned}
$$

which corresponds to selecting $(0,0),(2 \alpha, 0),(0,2 \beta),(2 \alpha, 2 \beta)$ as coincident frequencies. Motivated by the discussion in the previous section, we select the coincident frequencies

$$
\left(\sigma_{x}, \sigma_{y}\right)=\left(\alpha+d \cos \left(\frac{n}{2} \pi+\frac{1}{4} \pi\right), \beta+d \sin \left(\frac{n}{2} \pi+\frac{1}{4} \pi\right)\right), \quad 0 \leqq n \leqq 3,
$$

uniformly along the contour (4.17) and obtain the following stencil:

$$
L_{d,+}\left(E_{x}, E_{y}\right)=\frac{1}{h^{2}}\left[e^{\alpha h} E_{x}^{-1}+e^{-\alpha h} E_{x}+e^{\beta h} E_{y}^{-1}+e^{-\beta h} E_{y}-4 \cosh \left(\frac{d}{\sqrt{2}} h\right)\right]
$$

The multiplication of $E_{x}$ by the factor $e^{-\alpha h}$ in the $x$-direction of the space domain corresponds to a shift in the $s_{x}$-coordinate in the transform domain, where $s_{x}$ becomes $s_{x}-\alpha$, and a similar argument applies also to the $y$-direction. Therefore, the above scheme in fact shifts the center $(\alpha, \beta)$ of the circle (4.17) back to the origin and treats it as the Helmholtz equation with radius $d$. The coincident frequencies for these three schemes are plotted in Fig. 3.

Although all schemes have an accuracy of $O\left(h^{2}\right)$, schemes (4.19) and (4.20) are always diagonally dominant while the conventional scheme (4.18) loses this property for large cell Reynolds numbers $\alpha h$ and $\beta h$. This is one major disadvantage associated with the conventional central difference scheme. To overcome the instability of the conventional central difference scheme, in the context of multigrid methods Brandt [9] proposed a double discretization method which uses two different discretization schemes. The stable but first-order accurate upwind differencing scheme is used for smoothing while the unstable but second-order accurate central differencing scheme is used for computing the residues transferred to the coarser grid. As a result, the combination of these two schemes gives a stable algorithm and a second-order accurate solution. However, since two conflicting difference schemes are used, there is no algebraic convergence for such a multigrid process [9]. Note that the mode-dependent schemes (4.19) and (4.20) are both stable and second-order accurate. We can apply either one for both relaxation and residue transfer in a multigrid process and the resulting multigrid method will converge algebraically. 


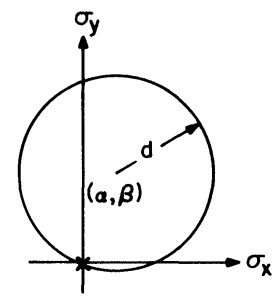

(a)

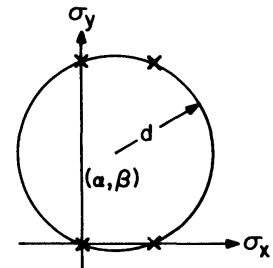

(b)

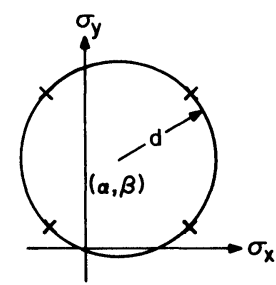

(c)

FIG. 3. Coincident frequencies of the (a) central difference, (b) Allen-Southwell, and (c) uniformly distributed mode-dependent 5-point stencil discretizations of the convection-diffusion equation.

Following a procedure similar to the one presented in $\S 4.2$, we can also design mode-dependent rotated 5-point and 9-point stencil discretization schemes for the convection-diffusion equation. This gives

$$
\begin{aligned}
& L_{d, \times}\left(E_{x}, E_{y}\right)=\frac{1}{2 h^{2}}\left[e^{(\alpha+\beta) h} E_{x}^{-1} E_{y}^{-1}+e^{(\alpha-\beta) h} E_{x}^{-1} E_{y}\right. \\
& \left.+e^{(-\alpha+\beta) h} E_{x} E_{y}^{-1}+e^{-(\alpha+\beta) h} E_{x} E_{y}-4 \cosh (d h)\right], \\
& L_{d, 9}\left(E_{x}, E_{y}\right)=\frac{\gamma_{\times}}{\gamma_{\times}+\gamma_{+}} L_{d,+}\left(E_{x}, E_{y}\right)+\frac{\gamma_{+}}{\gamma_{\times}+\gamma_{+}} L_{d, \times}\left(E_{x}, E_{y}\right)-\frac{\gamma_{\times} \gamma_{+}}{\gamma_{\times}+\gamma_{+}},
\end{aligned}
$$

with

$$
\begin{aligned}
& \gamma_{\times}=\frac{1}{h^{2}}[\cosh (\sqrt{2} d h)+1-2 \cosh (d h)], \\
& \gamma_{+}=\frac{1}{h^{2}}\left[2 \cosh (d h)+2-4 \cosh \left(\frac{d}{\sqrt{2}} h\right)\right] .
\end{aligned}
$$

These schemes have an accuracy of $O\left(h^{2}\right)$ and $O\left(h^{6}\right)$, respectively. Note that the constant $d=\sqrt{\alpha^{2}+\beta^{2}}$ in the convection-diffusion equation plays a similar role as the constant $|\lambda|$ in the Helmholtz equation. Therefore, as before, when $|\alpha|$ or $|\beta|$ becomes larger, the discretization schemes (4.20)-(4.22) become less accurate for a fixed $h$. 
5. Numerical examples. We use the one-dimensional and two-dimensional convection-diffusion equations as test problems to demonstrate the efficiency of the modedependent finite-difference method.

(1) One-dimensional test problem. Consider the one-dimensional convectiondiffusion equation on $[0,1]$

$$
\frac{d^{2} u}{d x^{2}}-a(x) \frac{d u}{d x}=0, \quad \text { where } a(x)=10+\varepsilon x+\frac{\varepsilon}{10+\varepsilon x}
$$

with given $u(0)$ and $u(1)$. Our goal is to study the effect of the linear perturbation term $\varepsilon x$ on the accuracy of the mode-dependent discretization scheme described in $\S 3$. Note that when $\varepsilon=0$, the coefficient $a(x)$ is constant, and according to our analysis we expect that in this case the mode-dependent discretization will be exact. The term $\varepsilon /(10+\varepsilon x)$ is added so that $(5.1)$ has the following analytic solution:

$$
u(x)=u(0)+[u(1)-u(0)] \frac{\exp \left(10 x+0.5 \varepsilon x^{2}\right)-1}{\exp (10+0.5 \varepsilon)-1}, \text { where } \exp (x)=e^{x} .
$$

The boundary conditions $u(0)=1$ and $u(1)=10$ are selected. We compare the conventional and mode-dependent central difference schemes, i.e.,

$$
\begin{gathered}
\left(1-\frac{a_{n} h}{2}\right) U_{n+1}-2 U_{n}+\left(1+\frac{a_{n} h}{2}\right) U_{n-1}=0, \\
\exp \left(-\frac{a_{n} h}{2}\right) U_{n+1}-2 \cosh \left(\frac{a_{n} h}{2}\right) U_{n}+\exp \left(\frac{a_{n} h}{2}\right) U_{n-1}=0,
\end{gathered}
$$

where $h=1 / N, 1 \leqq n \leqq N-1, U_{0}=u(0)$, and $U_{N}=u(1)$.

First, we study the effect of the grid size $h$ when the parameter $\varepsilon=1$. Figure 4 shows that the errors of both schemes are proportional to $O\left(h^{2}\right)$. Furthermore, for this choice of $\varepsilon$ and independent of the valve of $h$, the error of the mode-dependent scheme

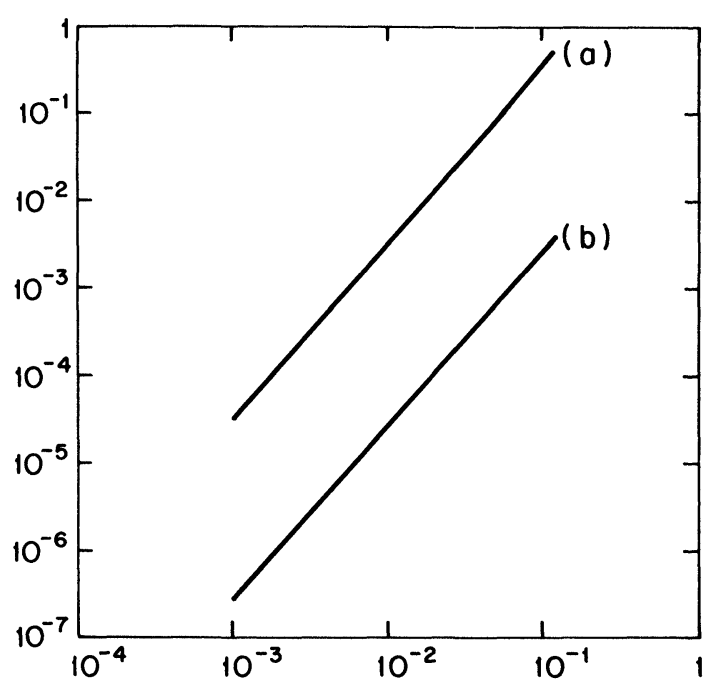

FIG. 4. $I_{x}$-norm of the error versus the grid size $h$ for (5.1) with $\varepsilon=1$ : (a) central difference scheme and (b) mode-dependent scheme. 
is approximately 100 times smaller than for the conventional scheme. Next, we study the impact on the error of variations of the coefficient function $a(x)$. The first derivative of the coefficient function $a(x)$ is approximately measured by the parameter $\varepsilon$, so that $\varepsilon$ can be used as a measure of the local variations of $a(x)$. Errors versus $\varepsilon$ for a fixed grid size $h=1 / 16$ are plotted in Fig. 5. From this figure, we see that the conventional scheme is insensitive to changes in $\varepsilon^{\prime}$, while the error of the mode-dependent scheme is proportional to $O(\varepsilon)$. Note that over the range of values of $\varepsilon$ that we consider, the error of the mode-dependent scheme is considerably smaller than for the conventional scheme. However, by extrapolating the two error curves in Fig. 5, it is clear that as $\varepsilon$ becomes very large, i.e., as the coefficient function $a(x)$ deviates drastically from its nominal value $a=10$, it will become preferable to use the conventional scheme. This suggests, as we would expect, that the mode-dependent discretization scheme should be employed only as long as we have at least an approximate knowledge of the modes appearing in the solution.

(2) Two-dimensional test problems. We consider two two-dimensional convectiondiffusion equations on $[0,1]^{2}$ with Dirichlet boundary conditions for which exact analytic solutions are already known.

\section{Example 1.}

$$
\frac{\partial^{2} u}{\partial x^{2}}+\frac{\partial^{2} u}{\partial y^{2}}-\left(8+\varepsilon_{x} x+\frac{\varepsilon_{x}}{8+\varepsilon_{x} x}\right) \frac{\partial u}{\partial x}-\left(6+\varepsilon_{y} y+\frac{\varepsilon_{y}}{6+\varepsilon_{y} y}\right) \frac{\partial u}{\partial y}=0,
$$

with exact solutions

(1a) $\left.\varepsilon_{x}=\varepsilon_{y}=0, \quad u(x, y)=\left(0.2+6 e^{8 x}\right)(0.01)+2 e^{6 y}\right)$

(1b) $\varepsilon_{x}=\varepsilon_{y}=0.002$,

$$
u(x, y)=\left[0.2+6 \exp \left(8 x+10^{-3} x^{2}\right)\right]\left[0.01+2 \exp \left(6 y+10^{-3} y^{2}\right)\right]
$$

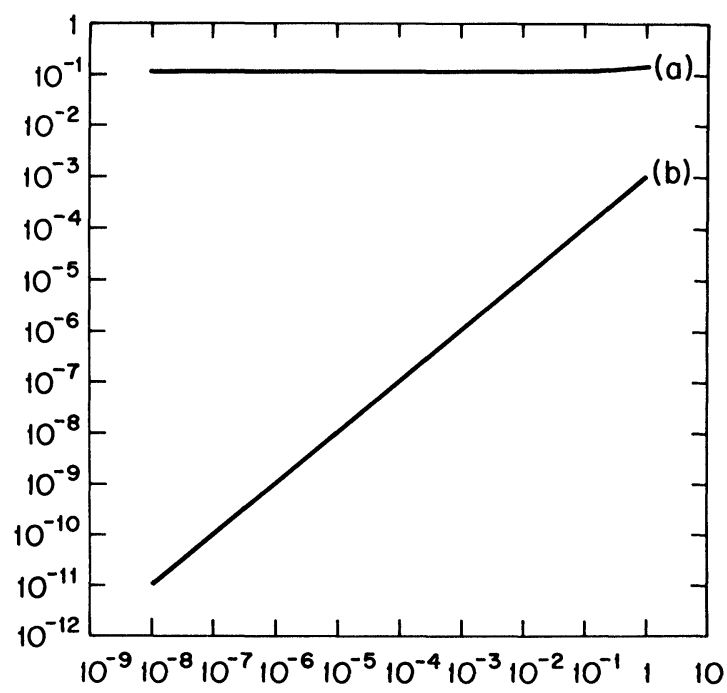

FIG. 5. $l_{\infty}$-norm of the error versus the parameter $\varepsilon$ for (5.1) with $h=\frac{1}{16}$ : (a) central difference scheme and (b) mode-dependent scheme. 
(1c) $\varepsilon_{x}=\varepsilon_{y}=0.02$,

$$
u(x, y)=\left[0.2+6 \exp \left(8 x+10^{-2} x^{2}\right)\right]\left[0.01+2 \exp \left(6 y+10^{-2} y^{2}\right)\right] .
$$

Example 2.

$$
\frac{\partial^{2} u}{\partial x^{2}}+\frac{\partial^{2} u}{\partial y^{2}}-\left(8+\frac{2 \varepsilon\left(\sigma_{1}-4\right)}{(1+\varepsilon x) \sigma_{1}+\varepsilon}\right) \frac{\partial u}{\partial x}-6 \frac{\partial u}{\partial y}=0,
$$

with exact solutions

$$
\begin{aligned}
& \text { (2a) } \varepsilon=0, \quad u(x, y)=10 \exp \left[\sigma_{1} x+\sigma_{2} y\right] \text {, } \\
& \text { (2b) } \varepsilon=0.01, \quad u(x, y)=(1+0.01 x) 10 \exp \left[\sigma_{1} x+\sigma_{2} y\right] \text {, } \\
& \text { (2c) } \varepsilon=0.1, \quad u(x, y)=(1+0.1 x) 10 \exp \left[\sigma_{1} x+\sigma_{2} y\right] \text {, }
\end{aligned}
$$

and where $\sigma_{1}=4+5 \cos (15 \pi / 16)$ and $\sigma_{2}=3+5 \sin (15 \pi / 16)$. We use the finitedifference schemes (4.18)-(4.22) discussed in $\S 4.3$ to discretize (5.3) and (5.5) with grid size $h=\frac{1}{4}, \frac{1}{8}, \frac{1}{16}$, and $\frac{1}{32}$. The resulting systems of equations are solved by the SOR method for test cases (1a) and (2a) and by a local relaxation method described in [8], [27], and [28] for test cases (1b), (1c), (2b), and (2c). We plot the errors versus the grid size in Figs. 6-11.

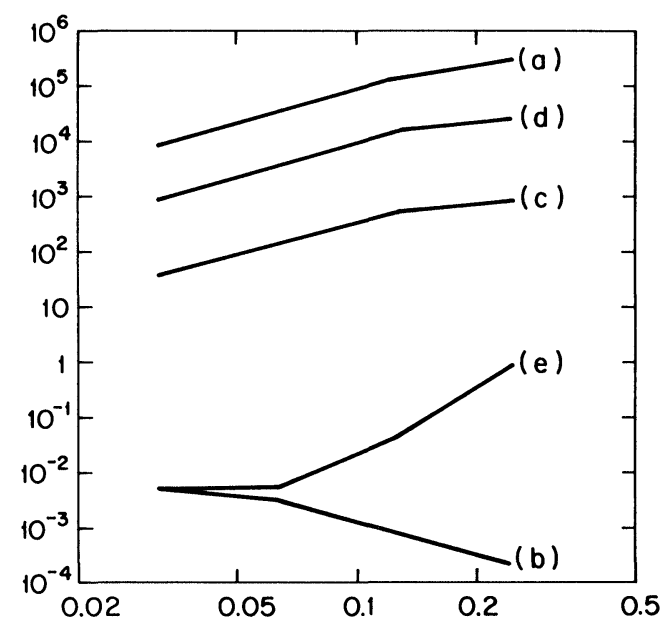

FIG. 6. $l_{\infty}$-norm of the error versus the grid size $h$ for $(5.4 \mathrm{a})$ : (a) $L_{d, c}$, (b) $L_{d, A S}$, (c) $L_{d,+}$, (d) $L_{d, \times}$, and (e) $L_{d, 9}$ given by (4.18)-(4.22).

For test case (1a), the solution contains four modes $1, e^{8 x}, e^{6 y}$, and $e^{8 x+6 y}$. In this case, since the Allen-Southwell scheme catches all these modes, it should be an exact method. Thus, the error plotted in Fig. 6 represents the numerical rounding error instead of the discretization error. The other 5-point stencil discretizations give an error proportional to $O\left(h^{2}\right)$ for fine grids. The 9-point stencil scheme is considerably more accurate than the other 5-point stencil schemes. It comes close to the exact method when the grid size is $\frac{1}{32}$.

Test case (1b) can be viewed as obtained from test case (1a) by introducing small linear perturbation terms $\varepsilon_{x} x$ and $\varepsilon_{y} y$ with $\varepsilon_{x}=\varepsilon_{y}=0.002$ in the coefficient functions. The Allen-Southwell scheme is not exact any longer, but still has a high accuracy. The 9-point discretization scheme has almost the same performance as the AllenSouthwell scheme. However, if we compare Figs. 6 and 7, we see that the coefficient 


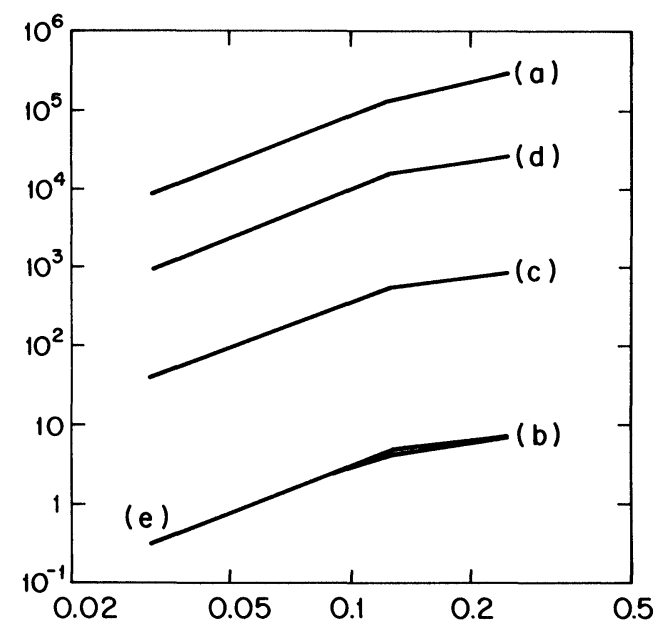

FIG. 7. $l_{\infty}$-norm of the error versus the grid size $h$ for $(5.4 \mathrm{~b})$ : (a) $L_{d, c}$, (b) $L_{d, A S}$, (c) $L_{d,+}$, (d) $L_{d, \times}$, and (e) $L_{d, 9}$ given by (4.18)-(4.22).

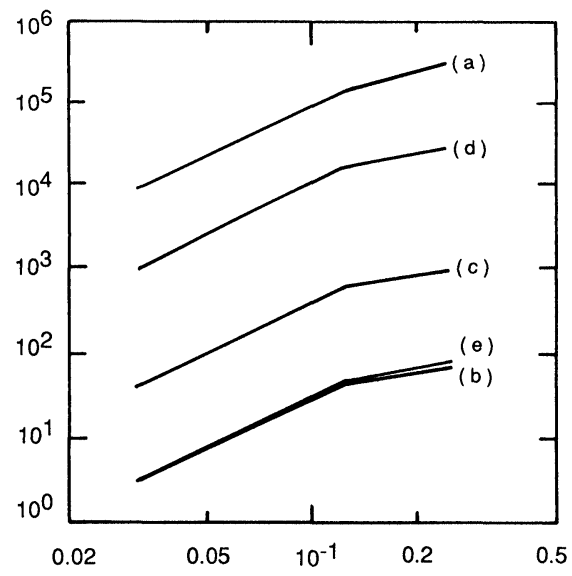

FIG. 8. $l_{\infty}$-norm of the error versus the grid size $h$ for (5.4c): (a) $L_{d, c}$, (b) $L_{d, A S}$, (c) $L_{d,+}$, (d) $L_{d, \times}$, and (e) $L_{d, 9}$ given by (4.18)-(4.22).

variations due to $\varepsilon_{x}$ and $\varepsilon_{y}$ make the error of the 9-point scheme 10 times as large as for the unperturbed case depicted in Fig. 6. The accuracy of the other 5-point stencil schemes remains approximately the same. When the magnitude of the perturbation terms is increased further as in test case (1c), the 9-point scheme and the AllenSouthwell scheme become less accurate than for test case (1b), as indicated by Fig. 8 .

For test case ( $2 \mathrm{a})$, the solution contains a single mode. All 5-point stencil discretization schemes have an accuracy of $O\left(h^{2}\right)$. The 9-point stencil discretization has an accuracy close to $O\left(h^{6}\right)$ for coarse grid sizes. Figure 9 shows also that although all 5-point stencil discretization schemes have a similar accuracy, the rotated 5-point operator $L_{d, \times}\left(E_{x}, E_{y}\right)$ given by (4.21) is slightly more accurate than the Allen-Southwell scheme. As was noted earlier, this difference can be attributed to a different choice of coincident frequencies satisfying the characteristic equation (4.17). 


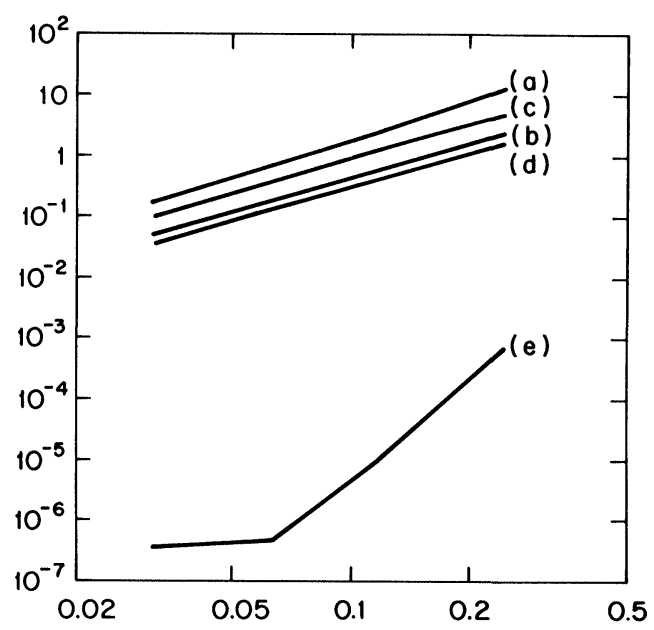

FIG. 9. $l_{\infty}$-norm of the error versus the grid size $h$ for (5.6a): (a) $L_{d, c}$, (b) $L_{d, A S}$, (c) $L_{d,+}$, (d) $L_{d, \times}$, and (e) $L_{d, 9}$ given by (4.18)-(4.22).

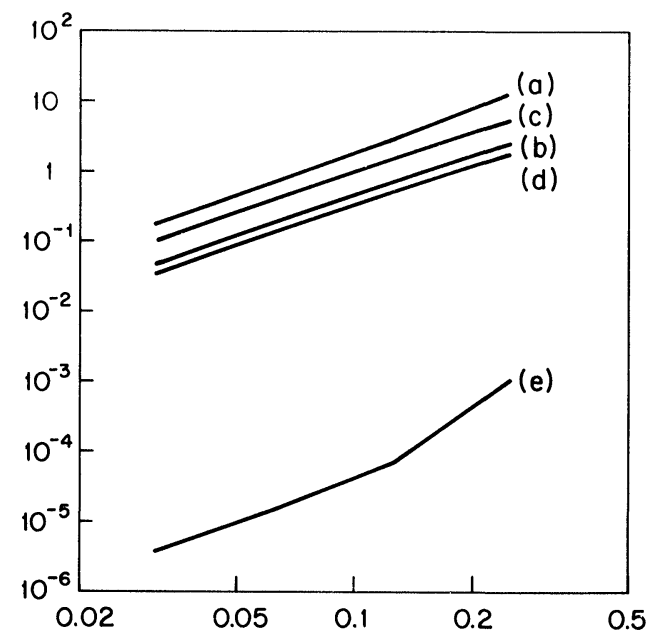

FIG. 10. $l_{\infty}$-norm of the error versus the grid size $h$ for (5.6b): (a) $L_{d, c}$, (b) $L_{d, A S}$, (c) $L_{d,+}$, (d) $L_{d, \times}$, and (e) $L_{d, 9}$ given by (4.18)-(4.22).

Test case (2b) is obtained by adding a small perturbation term to test case (2a). It appears that all 5-point stencil discretization schemes have a very similar accuracy in these two test cases. However, for the high-order 9-point stencil discretization, the perturbation makes the discretization error larger over fine grids. When $h=\frac{1}{32}$, the discretization error for the perturbed case (2b) is approximately 10 times larger than for the unperturbed case (2a). In test case (2c), we consider a larger perturbation effect. It turns out that the 9-point scheme behaves like a second-order accurate scheme; however, it is still more accurate than the 5-point discretization schemes.

From test cases (1b), (1c), (2b), and (2c), we may conclude that the modedependent discretization schemes still have a high accuracy for PDEs with nearly constant coefficients, but the 9-point stencil discretization is more sensitive to small variations of the coefficient functions than the 5-point stencil discretization schemes. 


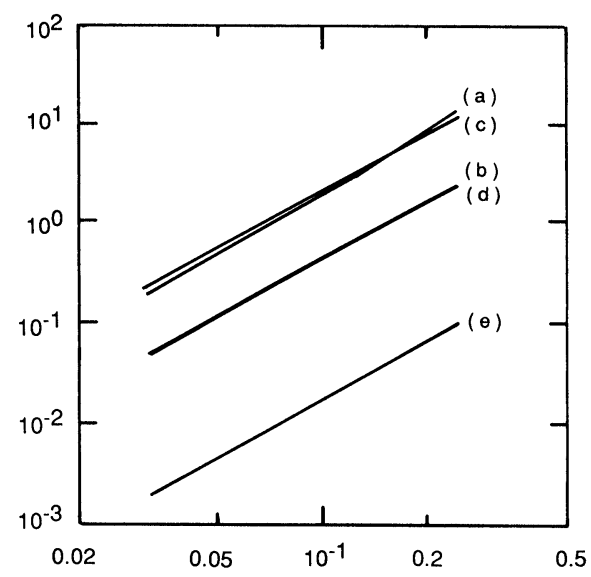

FIG. 11. $l_{\infty}$-norm of the error versus the grid size $h$ for (5.6c): (a) $L_{d, c}$, (b) $L_{d, A S}$, (c) $L_{d,+}$, (d) $L_{d, \times}$, and (e) $L_{d, 9}$ given by (4.18)-(4.22).

6. Related previous work. Although its properties were not always well understood, the mode-dependent finite-difference method has been discovered and rediscovered several times by a number of researchers and has been applied to the discretization of several types of ODEs and PDEs.

As was mentioned earlier, when the cell Reynolds number is large, the conventional central difference discretization of the convection-diffusion equation has convergence difficulties. Hence, the need for a mode-dependent scheme arises naturally when discretizing this equation, and more generally, when considering singular perturbation problems. Allen and Southwell [1] presented the first discretization of this type. A more detailed investigation of this scheme was performed by Dennis [13]. Since then, there have been a number of rediscoveries and elaborations such as [3], [10], [19], [29], [30], [36], [37], [40], and [41]. Applications of Allen-Southwell's scheme to two-dimensional or three-dimensional fluid flow problems can be found in [2], [14][18], [38], [39], and [42]. The methodology described in this paper can also be applied to the discretization of initial value ODEs. A mode-dependent finite-difference scheme for initial-value ODEs was first studied by Gautschi [20]. Some generalizations of Gautschi's work can be found in [5], [31], [35], [43], and [44].

As shown in $\S \S 3$ and 4 , the transform domain approach is a convenient tool to derive mode-dependent discretization schemes on a uniform grid. However, the modedependent concept is so general that it also applies to nonuniform grids. Some researchers have used the mode-dependent idea to design finite-element methods (see, e.g., [6], [11], [22]-[25], [34]).

Interestingly, the mode-dependent scheme has been introduced under a number of different names such as the locally exact technique [3], the weighted-mean scheme [19], the smart upwind method [21], the optimal finite analytic method [33], and the upstream-weighted difference scheme [36].

7. Conclusions and extensions. In this paper, we have used the spectral structure of differential operators to obtain more accurate finite-difference schemes. The transform domain point of view was shown to be simple and useful. For the case of homogeneous ODEs, we proposed a universal mode-dependent finite-difference scheme that is exact for constant-coefficient equations, and has a very high accuracy for equations with smoothly varying coefficients. For homogeneous PDEs, we considered 
mode-dependent 5-point, rotated 5-point, and 9-point stencil discretizations of the Laplace, Helmholtz, and convection-diffusion equations. The mode-dependent schemes for the Helmholtz and convection-diffusion equations turn out to be natural extensions of the schemes derived for the Laplace equation. We expect that the mode-dependent schemes that we have obtained will be quite useful for problems whose solution contain exponentially increasing or decreasing, or oscillatory components, provided that some very approximate information about these modes is available a priori. This information is usually revealed by the coefficients of the ordinary or partial differential equations of interest.

There exist similarities and differences between the mode-dependent finitedifference method and spectral methods. Both discretization techniques are based on a spectral analysis of the differential and difference operators and try to match their spectral properties. However, the spectral method analyzes spectra by using Fourier basis functions, i.e., functions with frequencies along the imaginary axis. In this approach, a large number of basis functions is usually required to synthesize a given function. Hence, in order to get a high degree of accuracy, more grid points are necessary and the resulting scheme is a global one. The mode-dependent finitedifference method enlarges the set of basis functions so that the spectral analysis can be performed in the entire transform domain. Since fewer basis functions are required to synthesize a function due to this enlargement, the resulting scheme is local. This local nature of the mode-dependent finite-difference method makes it easy to analyze and is insensitive to boundary conditions. In contrast, spectral methods are relatively more complicated and sensitive to different types of boundary conditions.

We basically focused on the discretization of a differential operator in the interior region and assumed the simplest Dirichlet boundary conditions throughout this paper. Since the finite-difference method is local, the discretization scheme for grid points in the interior region will not be affected by the specific nature of the boundary conditions. However, grid points along the boundary need some special treatment. Although the general mode-dependent concept should still apply in this case, some details need to be examined in later work. In addition, as mentioned above, it would be of interest to find a general procedure for estimating the error of mode-dependent finite-difference schemes when they are applied to varying-coefficient differential equations. Finally, it would also be interesting to study the application of the mode-dependent concept to the discretization of time-dependent partial differential equations.

Acknowledgment. The authors thank Professor Lloyd N. Trefethen for his encouragement and valuable discussions and the referees for their help in improving the technical presentation of this paper.

\section{REFERENCES}

[1] D. N. De G. Allen And R. V. Southwell, Relaxation methods applied to determine the motion, in two dimensions, of a viscous fluid past a fixed cylinder, Quart. J. Mech. Appl. Math., 8 (1955), pp. 129-145.

[2] D. N. De G. Allen, A suggested approach to finite-difference representation of differential equations, with an application to determine temperature-distributions near a sliding contact, Quart. J. Mech. Appl. Math., 15 (1962), pp. 11-33.

[3] K. E. BARRETT, The numerical solution of singular-perturbation boundary-value problems, Quart. J. Mech. Appl. Math., 27 (1974), pp. 57-68.

[4] A. E. Berger, J. M. Solomon, M. Ciment, S. H. Leventhal, And B. C. Weinberg, Generalized OCI schemes for boundary layer problems, Math. Comp., 35 (1980), pp. 695-731. 
[5] D. G. BETTIS, Numerical integration of products of Fourier and ordinary polynomials, Numer. Math., 14 (1970), pp. 421-434.

[6] W. S. BlACKBURN, Letters to the editor, Internat. J. Numer. Methods Engrg., 10 (1976), pp. 718-719.

[7] R. F. BOISVERT, Families of high order accurate discretizations of some elliptic problems, SIAM J. Sci. Statist. Comput, 2 (1981), pp. 268-284.

[8] E. F. Botta AND A. E. P. Veldman, On local relaxation methods and their application to convectiondiffusion equations, J. Comput. Phys., 48 (1981), pp. 127-149.

[9] A. BRANDT, Guide to multigrid development, in Multigrid Methods, W. Hackbusch and U. Trottenberg, eds., 1982, pp. 220-312, Springer-Verlag, New York.

[10] D. G. BRiggs, A finite difference scheme for the incompressible advection-diffusion equation, Comput. Methods Appl. Mech. Engrg., 6 (1975), pp. 233-241.

[11] I. Christie, D. F. Griffiths, A. R. Mitchell, And O. C. Zienkiewicz, Finite element methods for second order differential equations with significant first derivatives, Internat. J. Numer. Methods Engrg., 10 (1976), pp. 1389-1396.

[12] G. Dahlquist, A. Björck, And N. Anderson, Numerical Methods, Prentice-Hall, Englewood Cliffs, NJ, 1974.

[13] S. C. R. DENNIS, Finite differences associated with second-order differential equations, Quart. J. Mech. and Appl. Math., 13 (1960), pp. 487-507.

[14] - The numerical solution of the vorticity transport equation, in Proc. 3rd International Conference on Numerical Methods in Fluid Mechanics, 1973, pp. 120-129, Springer-Verlag, New York.

[15] S. C. R. DENnis, D. B. INGHAM, AND R. N. COOK, Finite-difference methods for calculating steady incompressible flows in three dimensions, J. Comput. Phys., 33 (1979), pp. 325-339.

[16] S. C. R. DENNIS, D. B. INGHAM, AND S. N. Singh, The steady flow of a viscous fluid due to a rotating sphere, Quart. J. Mech. Appl. Math., 34 (1981), pp. 361-381.

[17] - The slow translation of a sphere in a rotating viscous fluid, J. Fluid Mech., 117 (1982), pp. 251-267.

[18] T. M. El-MistiKAWY AND M. J. WERLE, Numerical method for boundary layers with blowing-the exponential box scheme, AIAA J., 16 (1978), pp. 749-751.

[19] M. E. FIADEIRO AND G. VERONIS, On weighted-mean schemes for the finite-difference approximation to the advection-diffusion equation, Tellus, 29 (1977), pp. 512-522.

[20] W. GAUTSCHI, Numerical integration of ordinary differential equations based on trigonometric polynomials, Numer. Math., 3 (1961), pp. 381-397.

[21] P. M. GRESho AND R. L. LeE, Don't suppress the wiggles-they're telling you something, Comput. \& Fluids, 9 (1981), pp. 223-253.

[22] J. C. Heinrich, P. S. Huyakorn, O. C. Zienkiewicz, And A. R. Mitchell, An "upwind" finite element scheme for two-dimensional convection transport equations, Internat. J. Numer. Methods Engrg., 11 (1977), pp. 131-143.

[23] J. C. HeINRICH AND O. C. ZIENkIEwiCZ, Quadratic finite element schemes for two-dimensional convective-transport problems, Internat. J. Numer. Methods Engrg., 11 (1977), pp. 1831-1844.

[24] P. S. HuyAKorn, Solution of steady-state, convective transport equation using an upwind finite element scheme, Appl. Math. Modelling, 1 (1977), pp. 187-195.

[25] A. KanARACHOS, Boundary layer refinements in convective diffusion problems, Internat. J. Numer. Methods Engrg., 18 (1982), pp. 167-180.

[26] L. V. Kantorovich AND V. I. Krylov, Approximate Methods of Higher Analysis, Interscience, New York, 1964.

[27] C.-C. J. KuO, B. C. Levy, AND B. R. Musicus, A local relaxation method for solving elliptic PDEs on mesh-connected arrays, SIAM J. Sci. Statist. Comput., 8 (1987), pp. 530-573.

[28] C.-C. J. KUO AND B. C. LEVY, A two-level four-color SOR method, SIAM J. Numer. Anal., to appear.

[29] B. P. LEONARD, A survey of finite differences with upwinding for numerical modelling of the incompressible convective diffusion equation, in Computational Techniques in Transient and Turbulent Flow, C. Taylor and K. Morgan, eds., Pineridge Press, Swansea, UK, 1981.

[30] W. LICK AND T. GASKInS, A consistent and accurate procedure for obtaining difference equations from differential equations, Internat. J. Numer. Methods Engrg., 20 (1984), pp. 1433-1441.

[31] T. LYCHE, Chebyshevian multistep methods for ordinary differential equations, Numer. Math., 19 (1972), pp. 65-75.

[32] R. E. LyNCH AND J. R. RICE, A high-order difference method for differential equations, Math. Comp., 34 (1980), pp. 333-372.

[33] R. Manohar And J. W. Stephenson, Optimal finite analytic methods, J. Heat Transfer, 104 (1982), pp. 432-437.

[34] A. Moult, D. Burley, And H. Rawson, The numerical solution of two-dimensional, steady flow problems by the finite element method, Internat. J. Numer. Methods Engrg., 14 (1979), pp. 11-35. 
[35] B. NETA AND C. H. FORD, Families of methods for ordinary differential equations based on trigonometric polynomials, J. Comput. Appl. Math., 10 (1984), pp. 33-38.

[36] G. D. RAITHBy AND K. E. TORRANCE, Upstream-weighted differencing schemes and their application to elliptic problems involving fluid flow, Comput. \& Fluids, 2 (1974), pp. 191-206.

[37] D. F. RosCOE, New methods for the derivation of stable difference representations for differential equations, J. Inst. Maths Appl., 16 (1975), pp. 291-301.

[38] - The solution of the three-dimensional Navier-Stokes equations using a new finite difference approach, Internat. J. Numer. Methods Engrg., 10 (1976), pp. 1299-1308.

[39] - The numerical solution of the Navier-Stokes equations for a three-dimensional laminar flow in curved pipes using finite-difference methods, J. Engrg. Math., 12 (1978), pp. 303-323.

[40] A. K. Runchal, Convergence and accuracy of three finite difference schemes for a two-dimensional conduction and convection problem, Internat. J. Numer. Methods Engrg., 4 (1972), pp. 541-550.

[41] D. B. Spalding, A novel finite difference formulation for differential expressions involving both first and second derivatives, Internat. J. Numer. Methods Engrg., 4 (1972), pp. 551-559.

[42] N. C. SteEle AND K. E. BARRETT, A 2nd order numerical method for laminar flow at moderate to high Reynolds numbers: entrance flow in a duct, Internat. J. Numer. Methods Engrg., 12 (1978), pp. 405-414.

[43] E. STIEFEl AND D. G. BetTis, Stabilization of Cowell's method, Numer. Math., 13 (1969), pp. $154-175$.

[44] P. J. VAN Der Houwen AND B. P. SommeiJer, Linear multistep methods with reduced truncation error for periodic initial-value problems, IMA J. Numer. Anal., 4 (1984), pp. 479-489. 